\title{
Genetic analysis of recently identified type 2 diabetes loci in 1,638 unselected patients with type 2 diabetes and 1,858 control participants from a Norwegian population-based cohort (the HUNT study)
}

\author{
J. K. Hertel • S. Johansson $\cdot$ H. Ræder $\cdot$ K. Midthjell • \\ V. Lyssenko • L. Groop • A. Molven • P. R. Njølstad
}

Received: 16 November 2007 / Accepted: 21 February 2008 / Published online: 24 April 2008

(C) Springer-Verlag 2008

\begin{abstract}
Aims/hypothesis Recent genome-wide association studies performed in selected patients and control participants have provided strong support for several new type 2 diabetes susceptibility loci. To get a better estimation of the true risk conferred by these novel loci, we tested a completely unselected population of type 2 diabetes patients from a Norwegian health survey (the HUNT study).

Methods We genotyped single nucleotide polymorphisms (SNPs) in PKN2, IGFBP2, FLJ39370 (also known as C4ORF32), CDKAL1, SLC30A8, CDKN2B, HHEX and FTO using a Norwegian population-based sample of 1,638 patients with type 2 diabetes and 1,858 non-diabetic control participants (the HUNT Study), for all of whom data on
\end{abstract}

Electronic supplementary material The online version of this article (doi:10.1007/s00125-008-0982-3) contains supplementary material, which is available to authorised users.

J. K. Hertel · S. Johansson · H. Ræder · P. R. Njølstad

Department of Clinical Medicine, University of Bergen,

Bergen, Norway

J. K. Hertel $\cdot$ S. Johansson

Center for Medical Genetics and Molecular Medicine,

Haukeland University Hospital,

Bergen, Norway

H. Ræder • P. R. Njølstad ( $\square)$

Department of Pediatrics, Haukeland University Hospital,

N-5021 Bergen, Norway

e-mail: pal.njolstad@uib.no

K. Midthjell

HUNT Research Center,

Department of Public Health and General Practice,

Norwegian University of Science and Technology,

Verdal, Norway
BMI, WHR, cholesterol and triacylglycerol levels were available. We used diabetes, measures of obesity and lipid values as phenotypes in case-control and quantitative association study designs.

Results We replicated the association with type 2 diabetes for rs 10811661 in the vicinity of $C D K N 2 B$ (OR 1.20, 95\% CI: 1.06-1.37, $p=0.004$ ), rs9939609 in FTO (OR 1.14, 95\% CI: $1.04-1.25, p=0.006)$ and rs 13266634 in SLC30A8 (OR $1.20,95 \%$ CI: $1.09-1.33, p=3.9 \times 10^{-4}$ ). We found borderline significant association for the IGFBP2 SNP rs4402960 (OR 1.10, 95\% CI: 0.99-1.22). Results for the HHEX SNP (rs1111875) and the CDKAL1 SNP (rs7756992) were non-significant, but the magnitude of effect was similar to previous estimates. We found no
V. Lyssenko • L. Groop

Department of Clinical Sciences, Diabetes and Endocrinology Research Unit, University Hospital Malmö, Lund University, Malmö, Sweden

L. Groop

Department of Medicine, Helsinki University Hospital, University of Helsinki,

Helsinki, Finland

A. Molven

The Gade Institute, University of Bergen,

Bergen, Norway

A. Molven

Department of Pathology, Haukeland University Hospital,

Bergen, Norway 
support for an association with the less consistently replicated FLJ39370 or PKN2 SNPs. In agreement with previous studies, FTO was most strongly associated with BMI $\left(p=8.4 \times 10^{-4}\right)$.

Conclusions/interpretation Our data show that SNPs near IGFBP2, CDKAL1, SLC30A8, CDKN2B, HHEX and FTO are also associated with diabetes in non-selected patients with type 2 diabetes.

Keywords Association study · BMI - GWA · Replication • Single nucleotide polymorphism · SNP. Susceptibility gene · Type 2 diabetes

$\begin{array}{ll}\text { Abbreviations } \\ \text { DGI } & \begin{array}{l}\text { Diabetes Genetics Initiative } \\ \text { FUSION }\end{array} \\ & \begin{array}{l}\text { Finland-United States Investigation of NIDDM } \\ \text { Genetics }\end{array} \\ \text { GWA } & \text { genome-wide association } \\ \text { HUNT } & \text { Nord-Trøndelag Health Study } \\ \text { HWE } & \begin{array}{l}\text { Hardy-Weinberg equilibrium } \\ \text { SNP }\end{array} \\ \text { single nucleotide polymorphism } \\ \text { WTCCC } & \text { Wellcome Trust Case Control Consortium }\end{array}$

\section{Introduction}

The worldwide rise in prevalence of type 2 diabetes has led to an intense search for genetic risk factors of type 2 diabetes. Although environmental influences certainly accelerate disease development in those with genetic predisposition, it is nonetheless of great clinical importance, and indeed a formidable challenge, to elucidate the genetic variants that increase the risk of type 2 diabetes [1]. In complex diseases such as type 2 diabetes, multiple genetic and environmental factors, as well as the interplay between these factors, determine the phenotype. Recently, several studies adopting a genome-wide association (GWA) strategy revealed genomic variants, each conferring a modest risk, to be implicated in type 2 diabetes [2-6]. The aim of the present study was to examine a Norwegian population (the NordTrøndelag Health Study [7]) for the association of loci with susceptibility for type 2 diabetes, obesity and lipid measures. Hence, this report describes an attempt to validate and expand results, based on studies adopting a GWA strategy, recently published by Sladek et al. [2], the Diabetes Genetics Initiative (DGI) [3], the Wellcome Trust Case Control Consortium (WTCCC)/UK Type 2 Diabetes Genetics Consortium collection [4], the Finland-United States Investigation of NIDDM Genetics (FUSION) [5] and the previous work by deCODE Genetics [6]. We also aimed to investigate whether a previously reported variant in FTO [8-10] was associated with risk for obesity in the Norwegian population.

\section{Methods}

Study participants and phenotype definitions The participants were $\geq 20$ years of age and drawn from an extensive population-based study (HUNT Study). The survey took place from 1995 to 1997 (HUNT2) in a Norwegian county with 127,000 inhabitants [7]. The county is representative of Norway as a whole with regard to the economy, industry and sources of income, age distribution, morbidity and mortality rates. The population is stable both ethnically and geographically, with less than $3 \%$ of people of non-white origin and a net emigration level of $0.3 \%$ per year (19962000), making it suitable for epidemiological studies. Of the 92,434 individuals considered eligible for the study, the participation rate was $71.3 \%$. The survey methods have been described in detail elsewhere [7, 11]. Diabetic participants were identified through a self-administered questionnaire, which has been shown to be a reliable source of information for epidemiological studies focusing on diabetes mellitus [12].

Genomic DNA was available for 1,850 diabetic participants, for 1,391 of whom more extensive clinical diabetes data were available. Participants with suspected type 1 diabetes were excluded from the study. Inclusion and exclusion criteria for the diabetic participants have been described previously [13]. In total, 1,638 type 2 diabetes participants and 1,858 non-diabetic participants (selfreported) were enrolled in the present study. The control participants were drawn from the same study population.

The study, which was approved by the Regional Committee for Research Ethics and the Norwegian Data Inspectorate, was performed according to the Helsinki Declaration, and all participants gave written informed consent.

Single nucleotide polymorphism selection The selection of single nucleotide polymorphisms (SNPs) prioritised for genotyping in the present study was based on publicly available GWA results as of June 2007, i.e. Sladek et al., the DGI-FUSION-WTCCC collaboration and deCODE Genetics [2-6, 8]. We included only SNPs that were robustly replicated in at least two of the GWA studies, i.e. IGFBP2, CDKAL1, SLC30A8, CDKN2B, HHEX and FTO. Because of the close relation between the Norwegian and Swedish populations, we also included two SNPs in $P K N 2$ and FLJ39370 (also known as C4ORF32), which had shown rather strong evidence of association in the DGI Scandinavian data set [3], although not in the UK sample sets [4].

Genotyping The genotyping was completed using MasSARRAY iPLEX system (Sequenom, San Diego, CA, USA). DNA from both patients and control participants 
were mixed on each 384-assay plate along with 20 internal controls and 12 blank samples uniquely distributed within each plate. Genotype concordance rate for the internal controls for the eight markers included was $>99.8 \%(n=$ 1,703 genotypes). We genotyped one 384-plate on two separate occasions and found a $100 \%$ concordance rate between the two runs ( $n=2,878$ duplicate genotypes). We successfully obtained genotypes from 3,496 individuals after removing 41 individuals due to low genotyping (maximum individual missingness rate $>0.2$ ), the total genotyping rate being $>98 \%$ in the remaining individuals.

Statistical analysis Each SNP was assessed for association with type 2 diabetes, BMI, WHR, and cholesterol and triacylglycerol levels using the software package PLINK [14]. The allelic association of each particular SNP with type 2 diabetes was examined using $\chi^{2}$ analysis. Further, we also applied a logistic regression model with age, sex and BMI as covariates. All SNPs were consistent with Hardy-Weinberg equilibrium (HWE), both in patients and control participants, and none of the markers were excluded on the basis of the HWE test $(p \leq 0.05)$. Estimates of the statistical power of our study, given different risk allele frequencies and ORs, are presented in Electronic supplementary material (ESM) Table 1 . We had $>80 \%$ power to detect high-frequency alleles with ORs of 1.15 to 1.20 , but only around $40 \%$ power if the true ORs were 1.10 . These estimates were performed using the Genetic Power Calculator [15].

For association analysis of BMI, WHR, and cholesterol and triacylglycerol levels as quantitative traits, we used a linear regression model using the PLINK software [14] both with and without age, sex and diabetic status as covariates. Before our analyses, we log-transformed triacylglycerol values for each participant in the cohort. Information on lipid-lowering therapy was not available and thus not considered.

Table 1 Clinical characteristics of the HUNT2 type 2 diabetes and control participants

\begin{tabular}{lcc}
\hline Variable & $\begin{array}{l}\text { Type 2 diabetes } \\
(n=1,638)\end{array}$ & $\begin{array}{l}\text { Control } \\
(n=1,858)\end{array}$ \\
\hline Sex (men:women; $n)$ & $764: 874$ & $880: 978$ \\
Age at diagnosis (years) & $58.4 \pm 15.1$ & - \\
Age at examination (years) & $68.4 \pm 12.1$ & $56.3 \pm 18.4$ \\
BMI (kg/m ${ }^{2}$ ) & $29.2 \pm 4.8$ & $26.5 \pm 4.2$ \\
Serum cholesterol (mmol/l) & $6.2 \pm 1.3$ & $6.1 \pm 1.3$ \\
Serum triacylglycerol (mmol/l) & $2.5 \pm 1.6$ & $1.9 \pm 1.2$ \\
Serum HDL-cholesterol (mmol/l) & $1.2 \pm 0.4$ & $1.4 \pm 0.4$ \\
Random serum glucose (mmol/l) & $9.6 \pm 4.2$ & $5.4 \pm 1.4$ \\
\hline
\end{tabular}

Values are means $\pm \mathrm{SD}$
Results for association of BMI with rs9939609 (FTO) genotypes were obtained by applying BMI as a continuous trait using a genotypic Cochran-Armitage trend test. These analyses were performed using Stata 8.0 (Stata Corp, College Station, TX, USA). The $p$ values presented in this report were not corrected for the number of tests performed and all CIs are presented as $95 \% \mathrm{CI}$.

\section{Results}

Results for the association of loci with type 2 diabetes The clinical characteristics of the participants enrolled in the present study are summarised in Table 1. Results for the association study using type 2 diabetes as phenotype are shown in Table 2. We found a significant association with type 2 diabetes for the SNPs rs 10811661 in the vicinity of $C D K N 2 B$ (OR 1.20, $p=0.004$ ), rs9939609 in FTO (OR $1.14, p=0.006$ ) and rs13266634 in SLC30A8 (OR 1.20, $p=$ $\left.3.9 \times 10^{-4}\right)$. Interestingly, the FTO variant, which was previously shown to be associated with diabetes probably via a primary effect on obesity [8], was still significant after adjustment for BMI (OR 1.14, $p=0.02)$.

We found a borderline association with type 2 diabetes for the IGFBP2 SNP rs4402960 (OR 1.10, $p=0.074$ ), which persisted also after controlling for cofactors. Furthermore, the HHEX SNP rs1111875 (OR 1.06, $p=0.196$ ) and the CDKAL1 SNP rs7756992 (OR 1.07, $p=0.192$ ) showed slightly lower ORs than in previous studies, and adjustment for age, sex and BMI increased the ORs only slightly. We found no association for rs17044137 (OR 1.01, $p=0.910$ ) and rs6698181 (OR 1.02, $p=0.690$ ) near FLJ39370 and $P K N 2$, respectively.

It is possible that some SNPs may have a more pronounced effect on type 2 diabetes in obese participants than in non-obese participants. We therefore stratified the patients and control participants on the basis of BMI, generating one group of participants with $\mathrm{BMI}<30 \mathrm{~kg} / \mathrm{m}^{2}$ and one with $\mathrm{BMI} \geq 30 \mathrm{~kg} / \mathrm{m}^{2}$ (ESM Tables 2 and 3 ). Consistent with the regression-based results (with BMI as cofactor), results were not significantly different between the obese and the non-obese groups. However, the statistical confidence of the association between the FTO SNP and type 2 diabetes was further increased (OR 1.27, $p=4.5 \times 10^{-4}$ ), when performing a case-control study involving obese type 2 diabetes participants and non-obese controls.

Association results for the obesity and lipid measures The results for the association study using BMI, WHR, and cholesterol and triacylglycerol levels as alternative phenotypes are shown in Table 3. The FTO rs9939609 was associated with BMI $\left(p=8.4 \times 10^{-4}\right)$, both for men $(p=5.1 \times$ 

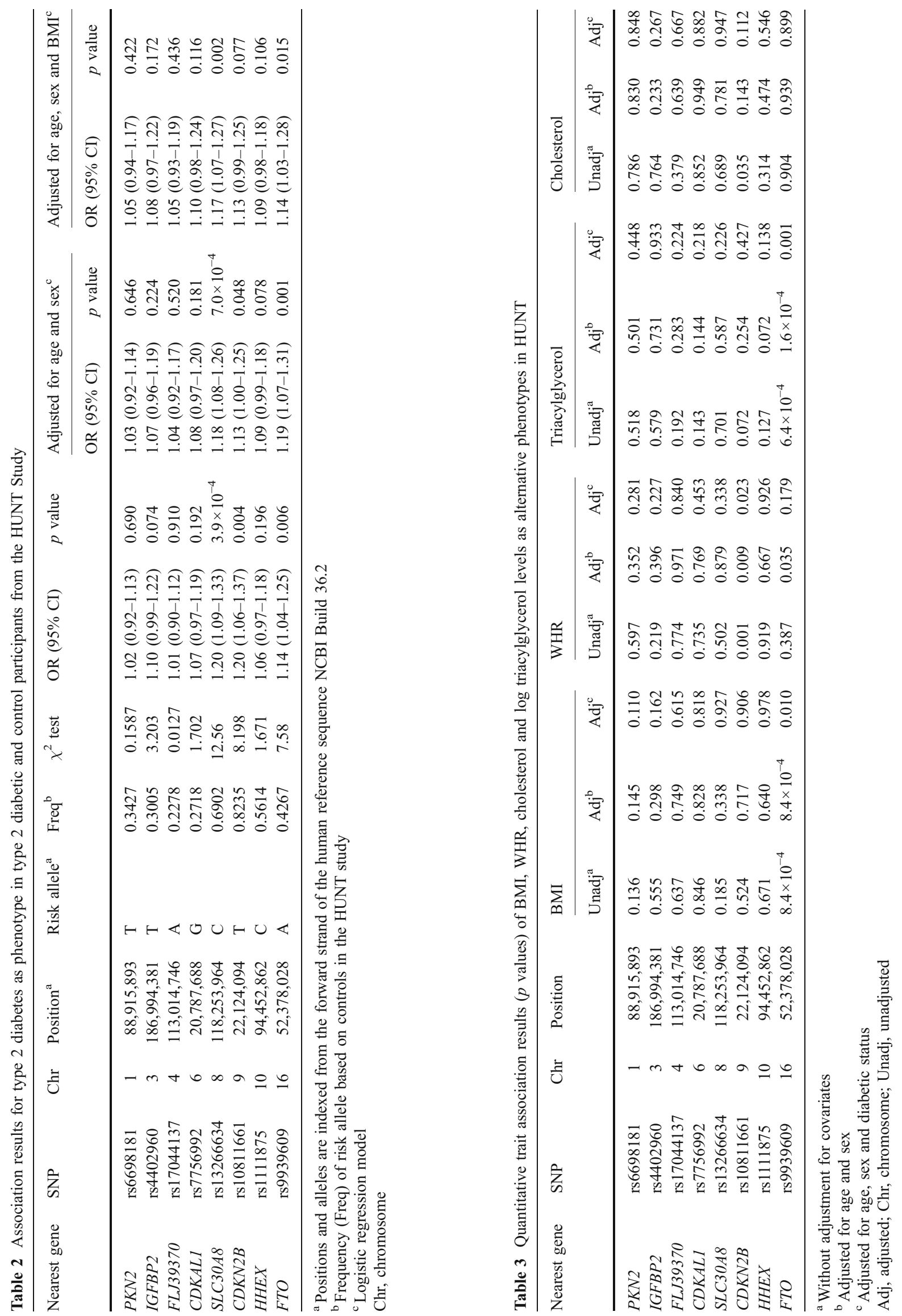
$10^{-4}$ ) and women ( $p=0.04$; ESM Table 4$)$. We also demonstrated a strong association with triacylglycerol levels for the FTO SNP $\left(p=1.4 \times 10^{-4}\right)$, which remained after controlling for age, sex and diabetes status.

Both BMI and WHR have been shown to be associated with type 2 diabetes, but from a clinical perspective, central obesity is suggested to generate a stronger type 2 diabetic risk than general obesity (BMI). Interestingly, the strong association with obesity shown for the FTO SNP using BMI as phenotype could not be demonstrated using WHR as a quantitative trait for obesity (Table 3 ). However, the SNP in the vicinity of $C D K N 2 B$ indicated association with WHR and also a nominal association with cholesterol. The SNPs in or near PKN2, IGFBP2, FLJ39370, CDKAL1, SLC30A8 and HHEX showed no association with quantitative metabolic traits in our study (Table 3).

\section{Discussion}

This is the first replication study of the recently identified type 2 diabetes risk variants using a large population-based body of material. The HUNT samples have been validated by genotyping of known type 2 diabetes risk variants in TCF7L2 and KCNJ11. We found a significant association with type 2 diabetes for the SNPs rs7903146 and rs12255372 in TCF7L2 and rs5219 in KCNJ11, similar to recent data (P. Thorsby, unpublished results) and with an OR similar to other studies $[16,17]$, indicating that the HUNT population contains a representative diabetes cohort and that our genotyping strategy was robust.

In the present study, we confirmed the diabetes association at the SLC30A8 locus. Our data were less compelling with regard to the SNPs tested near IGFBP2, HHEX and $C D K A L 1$. However, there was a trend in the same direction and of the same magnitude as in previous reports [2-6]. These genetic variants have been confirmed to be associated with type 2 diabetes [3-6], although in some cases with modest evidence in the initial stages and strengthened evidence only in combined analyses [5]. Our findings probably reflect the possibility that the associations are stronger in certain subgroups and that very large sample sizes are needed to formally replicate the IGFBP2, HHEX and $C D K A L 1$ data. It should also be emphasised that the risk variants have not been fine-mapped and that even subtle differences between different populations might affect linkage disequilibrium between test and disease variants.

We were able to confirm that the rs 10811661 located on chromosome $9 \mathrm{p}, 125 \mathrm{~kb}$ from the nearest gene $C D K N 2 B$, is associated with type 2 diabetes risk [3-5]. SNPs near this variant are associated with coronary artery disease [18-20].
In this regard it is interesting that we obtained evidence of nominal association between rs10811661 and both WHR and cholesterol levels. Thus, extensive studies of the region on chromosome 9 may provide more insight into the connections between type 2 diabetes and cardiovascular diseases.

The DGI defined two loci, FLJ39370 and PKN2, as interesting for follow-up studies [3], whereas the WTCCC and FUSION studies showed conflicting results $[4,5]$. We were not able to detect any association between type 2 diabetes and FLJ39370 or PKN2 candidate SNPs in the Norwegian sample, which is supposed to be genetically closely related to other Scandinavian populations. Hence, we suggest that these two SNPs probably represent false positive results in the DGI whole-genome scan.

As part of the WTCCC, Frayling et al. [8] reported that SNPs in the FTO gene region were highly associated with type 2 diabetes and BMI, suggesting that the FTO locus exerts its primary effect on adiposity and that it subsequently has an impact on type 2 diabetes [8]. In our study, we replicated the association between FTO and both an increased type 2 diabetes risk (OR 1.14 and $p=0.006$ ) and BMI $\left(p=8.4 \times 10^{-4}\right.$; Table 3$)$. Interestingly, the association between rs 9939609 and both type 2 diabetes and BMI remained significant after adjustment for BMI and diabetes status, respectively. It is also noteworthy that rs9939609 demonstrated a strong association with triacylglycerol levels $\left(p=1.4 \times 10^{-4}\right)$, which was not abolished after correcting for diabetes status. Thus, our data suggest that the relation between FTO and both BMI and diabetes is more complex than initially thought. Our results indicate that carriers of two A alleles at rs9939609 are at greater risk of being overweight, confirming results reported by Frayling et al. [8].

In contrast, using WHR as a quantitative measure of obesity, FTO showed only a nominal association after adjustment for sex and age, and no association when corrected for diabetes status. Moreover, the variant near $C D K N 2 B$, for which we observed no association with BMI, showed evidence of a nominal association with WHR. However, these results should be regarded as explorative; indeed, the lack of association between FTO and WHR in our sample could also be due to limited power. Larger studies are therefore needed to determine the role of FTO in central obesity.

Our study has both strengths and limitations. The HUNT study includes a well-characterised population from a clearly defined region of Norway where participants were recruited without regard to disease status and where the controls were drawn from the same population. Thus, there was no selection bias that could arise when conducting genetic studies [21]. Although we did not have the opportunity to formally test for possible population sub- 
structures with the limited numbers of markers genotyped, we believe that this population is more, rather than less homogeneous than other type 2 diabetes sample collections. Furthermore, the allele frequencies and the size effects are similar to previous publications, arguing against problems with population stratification. As a replication study, the sample size of $\sim 3,500$ patients and control participants was only powered to detect these relatively small ORs at a nominal significance. We argue that it is not necessary to correct for multiple comparisons when using diabetes as a trait, since this could be considered a pure replication study. However, for the additional phenotypic traits, results are more explorative and further studies are needed to address whether the observed additional associations with WHR and triacylglycerol levels represent true effects or spurious associations. Another point to note is that we tested only those SNPs that had been used in the initial reports $[2-6,8]$. Hence, another explanation for the lack of statistical support for some of the best GWA loci may be that the most highly associated SNPs vary from one population to another.

The whole-genome scan, in combination with large data sets, has recently shown its promise and delivered a whole set of new susceptibility loci for type 2 diabetes. The HUNT study with its unselected population may provide important insights, as it confirms most, but not all, previously identified loci associated with type 2 diabetes. Our results show that these findings can be generalised to a completely unselected population such as the Norwegian HUNT study (ESM Table 5). Importantly, the size effects that we observed are very similar to the estimates from the previous studies, indicating that the design of the first round of the whole-genome scans seems to pinpoint risk-alleles that show generality, at least in other Northern European populations. It is, nevertheless, crucial to continue to perform in-depth follow-up studies for these and future susceptibility loci in unselected samples of patients and control participants, since inclusion criteria based on age of onset, family history of the trait and BMI may affect the type of loci detected. Although speculative at this early stage, most loci detected so far seem to primarily affect insulin release rather than insulin resistance [22, 23]. However, before making such statements, researchers need to achieve a better understanding of how variation in the function of these loci leads to clinical disease.

Acknowledgements The University of Bergen, Haukeland University Hospital, Helse Vest, Innovest and Norwegian Research Council supported this study. Genotyping was in part provided by the CIGENE technology platform (Ås, Norway), which is supported by the Functional Genomics Programme (FUGE) of the Research Council of Norway. The Nord-Trøndelag Health
Study (HUNT) is a collaboration between the HUNT Research Center at the Norwegian University of Science and Technology, Verdal, the Norwegian Institute for Public Health and the NordTrøndelag County Council. The diabetes part of HUNT was partly supported by funds from GlaxoSmithKline Norway and the Norwegian Diabetes Association.

Duality of interest The authors declare that there is no duality of interest associated with this manuscript.

\section{References}

1. Permutt MA, Wasson J, Cox N (2005) Genetic epidemiology of diabetes. J Clin Invest 115:1431-1439

2. Sladek R, Rocheleau G, Rung J et al (2007) A genome-wide association study identifies novel risk loci for type 2 diabetes. Nature 445:881-885

3. Saxena R, Voight BF, Lyssenko V et al (2007) Genome-wide association analysis identifies loci for type 2 diabetes and triglyceride levels. Science 316:1331-1336

4. Zeggini E, Weedon MN, Lindgren CM et al (2007) Replication of genome-wide association signals in UK samples reveals risk loci for type 2 diabetes. Science 316:1336-1341

5. Scott LJ, Mohlke KL, Bonnycastle LL et al (2007) A genomewide association study of type 2 diabetes in Finns detects multiple susceptibility variants. Science 316:1341-1345

6. Steinthorsdottir V, Thorleifsson G, Reynisdottir I et al (2007) A variant in CDKAL1 influences insulin response and risk of type 2 diabetes. Nat Genet 39:770-775

7. Holmen J, Midthjell K, Krüger $\varnothing$ et al (2003) The NordTrøndelag Health Study 1995-97 (HUNT2): objectives, contents, methods and participation. Norw J Epidemiol 13:19-32

8. Frayling TM, Timpson NJ, Weedon MN et al (2007) A common variant in the FTO gene is associated with body mass index and predisposes to childhood and adult obesity. Science 316: 889-894

9. Dina C, Meyre D, Gallina S et al (2007) Variation in FTO contributes to childhood obesity and severe adult obesity. Nat Genet 39:724-726

10. Scuteri A, Sanna S, Chen WM et al (2007) Genome-wide association scan shows genetic variants in the FTO gene are associated with obesity-related traits. PLoS Genet 3:e115

11. Midthjell K, Krüger O, Holmen J et al (1999) Rapid changes in the prevalence of obesity and known diabetes in an adult Norwegian population. The Nord-Trøndelag Health Surveys: 1984-1986 and 1995-1997. Diabetes Care 22:1813-1820

12. Midthjell K, Holmen J, Bjørndal A et al (1992) Is questionnaire information valid in the study of a chronic disease such as diabetes? The Nord-Trøndelag diabetes study. J Epidemiol Community Health 46:537-542

13. Johansson S, Raeder H, Eide SA et al (2007) Studies in 3,523 Norwegians and meta-analysis in 11,571 subjects indicate that variants in the hepatocyte nuclear factor 4 alpha (HNF4A) P2 region are associated with type 2 diabetes in Scandinavians. Diabetes 56:3112-3117

14. Purcell S, Neale B, Todd-Brown K et al (2007) PLINK: a tool set for whole-genome association and population-based linkage analyses. Am J Hum Genet 81:559-575 
15. Purcell S, Cherny SS, Sham PC (2003) Genetic power calculator: design of linkage and association genetic mapping studies of complex traits. Bioinformatics 19:149-150

16. Grant SF, Thorleifsson G, Reynisdottir I et al (2006) Variant of transcription factor 7-like 2 (TCF7L2) gene confers risk of type 2 diabetes. Nat Genet 38:320-323

17. Florez JC, Burtt N, de Bakker PI et al (2004) Haplotype structure and genotype-phenotype correlations of the sulfonylurea receptor and the islet ATP-sensitive potassium channel gene region. Diabetes 53:1360-1368

18. Samani NJ, Erdmann J, Hall AS et al (2007) Genomewide association analysis of coronary artery disease. N Engl J Med 357:443-453

19. Helgadottir A, Thorleifsson G, Manolescu A et al (2007) A common variant on chromosome 9p21 affects the risk of myocardial infarction. Science 316:1491-1493
20. McPherson R, Pertsemlidis A, Kavaslar N et al (2007) A common allele on chromosome 9 associated with coronary heart disease. Science 316:1488-1491

21. De Silva NM, Steele A, Shields B et al (2007) The transcription factor 7-like 2 (TCF7L2) gene is associated with Type 2 diabetes in UK community-based cases, but the risk allele frequency is reduced compared with UK cases selected for genetic studies. Diabet Med 24:1067-1072

22. Lyssenko V, Lupi R, Marchetti P et al (2007) Mechanisms by which common variants in the TCF7L2 gene increase risk of type 2 diabetes. J Clin Invest 117:2155-2163

23. Grarup N, Rose CS, Andersson EA et al (2007) Studies of association of variants near the HHEX, CDKN2A/B, and IGF2BP2 genes with type 2 diabetes and impaired insulin release in 10,705 Danish subjects: validation and extension of genomewide association studies. Diabetes 56:3105-3111 